\title{
Phase diagram of two-color quark matter at nonzero baryon and isospin density
}

\author{
Jens O. Andersen, ${ }^{1, *}$ and Tomáš Brauner, \\ ${ }^{1}$ Department of Physics, Norwegian University of Science and Technology, Høgskoleringen 5, N-7491 Trondheim, Norway \\ ${ }^{2}$ Institut für Theoretische Physik, Goethe-Universität, \\ Max-von-Laue-Straße 1, D-60438 Frankfurt am Main, German声
}

\begin{abstract}
We investigate the properties of cold dense quark matter composed of two colors and two flavors of light quarks. In particular, we perform the first model calculation of the full phase diagram at nonzero baryon and isospin density, thus matching the model-independent predictions of chiral perturbation theory at low density to the conjectured phase structure at high density. We confirm the presence of the Fulde-Ferrell (FF) phase in the phase diagram and study its dependence on the tunable parameter in the Lagrangian that simulates the effects of the quantum axial anomaly. As a byproduct, we clarify the calculation of the thermodynamic potential in the presence of the FF pairing, which was previously based on an ad hoc subtraction of an unphysical cutoff artifact. Furthermore, we argue that close to the diquark (or pion) Bose-Einstein condensation transition, the system behaves as a dilute Bose gas so that our simple fermionic model in the mean-field approximation is not quantitatively adequate. We suggest that including thermal fluctuations of the order parameter for Bose-Einstein condensation is crucial for understanding available lattice data.
\end{abstract}

PACS numbers: 11.30.Qc, 12.39.Fe, 21.65.Qr, 74.81.-g

Keywords: two-color QCD; Nambu-Jona-Lasinio model; diquark condensation

\section{INTRODUCTION}

The attempts to understand the phase structure of quantum chromodynamics (QCD) together with the infamous sign problem at nonzero baryon chemical potential, have triggered interest in several QCD-like theories. These include QCD at nonzero isospin density [1], QCD with adjoint quarks and two-color QCD [2]. The common feature of these theories is that they are free of the sign problem. This on the one hand admits their straightforward simulation using lattice Monte-Carlo techniques and a check of these methods against model-independent predictions of chiral perturbation theory. On the other hand, it allows a direct test of various model approaches at high temperature and/or density where chiral perturbation theory is not applicable. Hence the study of QCD-like theories contributes to our understanding of the physics of strongly-coupled gauge theories at nonzero temperature and density.

In this paper we investigate the thermodynamics of two-color QCD, which has attracted most interest among the QCD-like theories. We focus on the case of two light quark flavors with nonzero baryon and isospin chemical potentials. Although the underlying mechanisms of confinement and chiral symmetry breaking in two-color QCD are believed to be the same as in the real, three-color $\mathrm{QCD}$, the actual physical behavior is quite different, especially at nonzero baryon density. First, two quarks can combine into a color singlet (leaving the gauge symmetry unbroken) which implies that at high density where

\footnotetext{
* jens.andersen@ntnu.no

† brauner@ujf.cas.cz

$\ddagger$ On leave from Department of Theoretical Physics, Nuclear Physics Institute ASCR, CZ-25068 Řež, Czech Republic.
}

Cooper pairing near the Fermi surface occurs, the system behaves as a baryon superfluid rather than a color superconductor. Second, even in the confined phase, the colorless baryons are formed by two quarks, which means that "nuclear" matter is actually a Bose gas and finite baryon density is realized through Bose-Einstein condensation (BEC).

The very fact that two-color QCD is free of the sign problem follows from the (pseudo)reality of the gauge group representation in which the quarks transform. This has another notable consequence: the Pauli-Gürsey symmetry [3, 4] connecting quarks with antiquarks. As a result, the spectrum of Nambu-Goldstone (NG) bosons of the spontaneously broken global flavor symmetry includes pseudoscalar mesons as well as diquarks in a single multiplet. In fact, it is the presence of light particles carrying baryon number that allows to study the phase diagram of two-color QCD at nonzero temperature and baryon density using the techniques of effective field theory $[2,[5,[6]$.

While chiral perturbation theory provides a modelindependent parameterization of all observables in terms of a few low-energy couplings, its validity is limited to the region of low density. Several approaches have therefore been used to extend its results. These include the linear sigma model [7], random matrix theory [8], or high-density effective theory [9]. The Nambu-JonaLasinio (NJL) model was first applied to two-color QCD in Ref. [10] for one quark flavor and in Ref. [11] for two flavors. In [12] the crossover from BEC of molecular diquarks to the Bardeen-Cooper-Schrieffer (BCS) pairing of quarks was studied. Nonzero isospin chemical potential imposes stress on this pairing and when sufficiently large, induces a first-order transition to the normal phase. Around this transition, a narrow window with inhomo- 
geneous pairing may exist, as conjectured in [5] and explicitly verified in [13]. Despite the number of papers on the subject pursuing different analytic approaches, the main body of work has been done on the lattice, using both the strong-coupling expansion [14] and Monte-Carlo simulations [15, 16].

The plan of the paper is as follows. In Sec. II we introduce the NJL model for two-color QCD and demonstrate how it can be derived in a way which makes the extended symmetry of two-color QCD manifest. Section [III is entirely devoted to the mean-field approximation within this model. The reason is that inclusion of the Fulde-Ferrell (FF) pairing brings nontrivial regularization issues, in particular in connection with the widely used sharp three-dimensional cutoff. Section IV presents the numerical results for the phase diagram at nonzero baryon and isospin density. We include chiral, diquark and pion condensates with the possibility of spatial (FF) modulation of the latter two. Using a generalized NJL interaction, we also allow for isospin breaking by different chiral condensates of the $u$ and $d$ quarks. We confirm the presence of the FF phase at high chemical potential, in agreement with [13]. We show that the region in the phase diagram where $\mathrm{FF}$ pairing occurs depends sensitively on the parameter in the Lagrangian that measures axial symmetry breaking by the quantum anomaly. In Sec. $\mathrm{V}$ we investigate the collective modes in the NJL model. We calculate their propagators and determine their masses and dissociation temperatures. In Sec. VI we discuss in some depth two-color quark matter at nonzero baryon and zero isospin density, which corresponds to the conditions being simulated on the lattice. Recent progress in the lattice computations led to first attempts to probe BEC of diquarks and the region of moderate baryon density [16]. One of the motivations for the present work was an attempt to understand the available lattice data on the thermodynamics of cold and dense two-color quark matter. Even though we have not been able to demonstrate quantitative agreement between the lattice simulation and model calculations, we outline a possible direction along which it can presumably be reached. Finally, in Sec. VII we summarize and conclude.

\section{NJL LAGRANGIANS FOR TWO-COLOR QUARK MATTER}

In any model attempting to mimic some features of full QCD, it is mandatory that its Lagrangian has the same symmetries as the underlying theory. In 11] the NJL interaction for two-color QCD was derived by a Fierz transformation of the four-quark interaction inspired by onegluon exchange, in which the flavor symmetry is manifest. Here we introduce a formalism which makes it possible to construct NJL interactions with desired symmetry properties directly. Our discussion will parallel that of Kogut et al. [2]. Yet we believe it is worthwhile to show some details, since instead of working with the Weyl spinors for the quark fields as in [2], we keep the Dirac spinors and introduce the Nambu doublet notation, as is common in the literature on the NJL model.

Our starting point is the Lagrangian for the quark sector of two-color QCD,

$$
\mathscr{L}_{2 \mathrm{cQCD}}=\bar{\psi} i \not D \psi-m_{0} \bar{\psi} \psi,
$$

where $D_{\mu} \psi=\left(\partial_{\mu}-i g \sigma_{a} A_{\mu}^{a}\right) \psi$ is the color-SU(2) covariant derivative, $m_{0}$ is the current mass common to all quark flavors, and the flavor indices are suppressed. Next we replace the usual Dirac spinor, consisting of the left- and right-handed components, with the purely left-handed Nambu spinor and its (conveniently defined) charge conjugate,

$$
\Psi=\left(\begin{array}{c}
\psi_{L} \\
\sigma_{2} \psi_{R}^{\mathcal{C}}
\end{array}\right), \quad \Psi^{\mathcal{C}}=\sigma_{2}\left(\begin{array}{c}
\psi_{L}^{\mathcal{C}} \\
\left(\sigma_{2} \psi_{R}^{\mathcal{C}}\right)^{\mathcal{C}}
\end{array}\right)=\left(\begin{array}{c}
\sigma_{2} \psi_{L}^{\mathcal{C}} \\
-\psi_{R}
\end{array}\right),
$$

where $\psi^{\mathcal{C}}=C \bar{\psi}^{T}$ is usual Dirac charge conjugation. The two-color QCD Lagrangian (11) can then be rewritten in the block-matrix form

$$
\mathscr{L}_{2 \mathrm{cQCD}}=\bar{\Psi} i \not D \Psi-\left[\frac{1}{2} m_{0} \overline{\Psi^{\mathcal{C}}}\left(\begin{array}{cc}
0 & \mathbb{1} \\
-\mathbb{1} & 0
\end{array}\right) \Psi+\text { H.c. }\right] .
$$

All entries of the $2 \times 2$ Nambu mass matrix are themselves matrices in flavor space. Without going into details which are covered in literature [2], we note that thanks to the (pseudo)reality of the gauge-SU(2) generators, $\mathscr{L}_{2 \mathrm{cQCD}}$ with $N_{f}$ quark flavors has an apparent $\mathrm{SU}\left(2 N_{f}\right)$ symmetry in the chiral limit. The baryon number, being generated by

$$
B=\frac{1}{2}\left(\begin{array}{cc}
\mathbb{1} & 0 \\
0 & -\mathbb{1}
\end{array}\right),
$$

is incorporated as a part of this simple group, while a mere change of the phase of $\Psi$ corresponds to axial $\mathrm{U}(1)_{\mathrm{A}}$ transformations. This symmetry is known to be broken at the quantum level by the axial anomaly.

As is clear from Eq. (2), the mass operator in the Nambu notation is proportional to

$$
\overline{\Psi^{\mathcal{C}}} \Sigma_{1} \Psi=\Psi^{T} C \sigma_{2} \Sigma_{1} \Psi, \quad \Sigma_{1}=\left(\begin{array}{cc}
0 & -\mathbb{1} \\
\mathbb{1} & 0
\end{array}\right) .
$$

The standard chiral condensate which represents the ground state in the vacuum has the same flavor structure as the mass term. Therefore, the multiplet of mesons, one of which is to condense in the ground state and the others to become NG modes, transform as a rank-2 antisymmetric tensor of $\mathrm{SU}\left(2 N_{f}\right)$. For $N_{f}=2$ we make use of the local group isomorphism $\mathrm{SU}(4) \simeq \mathrm{SO}(6)$ to conclude that with a suitable basis of $4 \times 4$ antisymmetric matrices, $\vec{\Sigma}=\left\{\Sigma_{i}\right\}_{i=1}^{6}$, the operator $\overline{\Psi^{\mathcal{C}}} \vec{\Sigma} \Psi$ transforms as a complex vector of $\mathrm{SO}(6)$. The basis matrices are unitary and satisfy the orthogonality condition $\Sigma_{i} \Sigma_{j}^{\dagger}+\Sigma_{j} \Sigma_{i}^{\dagger}=2 \delta_{i j}$. They may be chosen to carry the quantum numbers of 
the individual meson modes: three pions, sigma, diquark and the antidiquark. The explicit form of the matrices with these properties was given in 17].

It is now obvious how to construct a NJL interaction Lagrangian with $\mathrm{SO}(6)$ symmetry. As long as we only consider the Lorentz-scalar bilinear operator $\overline{\Psi^{\mathcal{C}}} \vec{\Sigma} \Psi$, thus selecting bosonic modes with specific quantum numbers [18], there are only two ways to make an $\mathrm{SO}(6)$ invariant out of the vector $\overline{\Psi^{\mathcal{C}}} \vec{\Sigma} \Psi$ : taking a scalar product with itself or its complex conjugate,

$$
\begin{aligned}
\mathscr{L}_{\mathrm{U}(4)} & =G\left|\overline{\Psi^{\mathcal{C}}} \vec{\Sigma} \Psi\right|^{2} \\
\mathscr{L}_{\mathrm{SU}(4)} & =\frac{G}{2}\left[\left(\overline{\Psi^{\mathcal{C}}} \vec{\Sigma} \Psi\right)^{2}+\text { H.c. }\right] .
\end{aligned}
$$

The subscripts emphasize the important fact that while $\mathscr{L}_{\mathrm{U}(4)}$ is invariant under the $\mathrm{U}(1)_{\text {A }}$ group of phase transformations of $\Psi, \mathscr{L}_{\mathrm{SU}(4)}$ obviously breaks it. Inserting explicit expressions for the $\Sigma_{i}$ matrices, these two interactions can be readily rewritten in terms of the original Dirac spinor $\psi$. It is convenient to work with the following combinations,

$$
\begin{aligned}
\mathscr{L}_{1} & =\frac{1}{2}\left[\mathscr{L}_{\mathrm{U}(4)}+\mathscr{L}_{\mathrm{SU}(4)}\right] \\
& =G\left[(\bar{\psi} \psi)^{2}+\left(\bar{\psi} i \gamma_{5} \vec{\tau} \psi\right)^{2}+\left|\overline{\psi^{\mathcal{C}}} \gamma_{5} \sigma_{2} \tau_{2} \psi\right|^{2}\right], \\
\mathscr{L}_{2} & =\frac{1}{2}\left[\mathscr{L}_{\mathrm{U}(4)}-\mathscr{L}_{\mathrm{SU}(4)}\right] \\
& =G\left[\left(\bar{\psi} i \gamma_{5} \psi\right)^{2}+(\bar{\psi} \vec{\tau} \psi)^{2}+\left|\overline{\psi^{\mathcal{C}}} \sigma_{2} \tau_{2} \psi\right|^{2}\right],
\end{aligned}
$$

where $\vec{\tau}$ are Pauli matrices in isospin space. The quantum numbers of the various channels are now apparent: $\mathscr{L}_{1}$ contains the sigma, pions and the scalar (anti)diquark, while $\mathscr{L}_{2}$ contains their parity partners.

Note that $\mathscr{L}_{1}$ is essentially the minimal two-flavor NJL Lagrangian [19], augmented by a diquark term. The only difference with Lagrangians used in model investigations of dense (three-color) quark matter is that here the $\mathrm{SU}(4)$ symmetry fixes the coupling in the diquark channel to be the same as for the meson terms. This is the Lagrangian used in [11, 12]. However, since we have two independent interaction terms in Eq. (3), the most general interaction one can construct is a linear combination

$$
\mathscr{L}_{\text {int }}=\mathscr{L}_{1}+\zeta \mathscr{L}_{2},
$$

and was written down for the first time in [20]. At $\zeta=1$ the Lagrangian reduces to $\mathscr{L}_{\mathrm{U}(4)}$, preserving the axial $\mathrm{U}(1)_{\mathrm{A}}$, and is a direct descendant of the current-current interaction used in 11]. The case of $\zeta=-1$, that is, $\mathscr{L}_{\mathrm{SU}(4)}$, corresponds to the two-flavor instanton-induced interaction, breaking $\mathrm{U}(1)_{\mathrm{A}}$ maximally [4]. In the present paper $\zeta$ is treated as a free parameter, the only restriction being $\zeta \leq 1$. This follows from the fact that in the vacuum, the scalar chiral condensate must be preferred over the pseudoscalar one, as guaranteed by the Vafa-Witten theorem [21]. We note in passing that in the threecolor NJL model, one usually introduces the parameter $\alpha=(1-\zeta) / 2$ which measures explicit $\mathrm{U}(1)_{\mathrm{A}}$ breaking by the axial anomaly so that the interaction Lagrangian can also be expressed as $\mathscr{L}_{\text {int }}=(1-\alpha) \mathscr{L}_{\mathrm{U}(4)}+\alpha \mathscr{L}_{\mathrm{SU}(4)}$. The typical values of $\alpha$ lie somewhere in the range $0.1-0.2$ [22].

The full NJL Lagrangian now reads

$$
\mathscr{L}=\bar{\psi}\left[i \not \partial-m_{0}+\frac{1}{2} \gamma_{0}\left(\mu_{B}+\tau_{3} \mu_{I}\right)\right] \psi+\mathscr{L}_{\text {int }},
$$

where $\mu_{B}$ and $\mu_{I}$ are baryon number and isospin chemical potentials, both being assumed positive without lack of generality. In [20] this Lagrangian with the generalized interaction (4) was employed to study the masses of the scalar mesons and hence the axial symmetry restoration in the meson spectrum. Here it will be crucial at high baryon and isospin chemical potentials, for it allows to introduce different $u$-quark and $d$-quark chiral condensates within the mean-field approximation.

\section{MEAN-FIELD APPROXIMATION}

As mentioned above, the scalar chiral and diquark condensates are preferred to the pseudoscalar ones. Since we will not be interested in the corresponding non-NG modes (eta meson and the pseudoscalar diquark) either, we will completely neglect the interaction terms in the $\bar{\psi} i \gamma_{5} \psi$ and $\overline{\psi^{\mathcal{C}}} \sigma_{2} \tau_{2} \psi$ channels. The remaining four channels can be rewritten using the Hubbard-Stratonovich transformation. The Lagrangian can then be written as

$$
\begin{gathered}
\mathscr{L}=\bar{\psi}\left[i \not \partial-m_{0}+\frac{1}{2} \gamma_{0}\left(\mu_{B}+\tau_{3} \mu_{I}\right)-\sigma-i \gamma_{5} \vec{\tau} \cdot \vec{\pi}-\zeta \vec{\tau} \cdot \vec{\rho}\right] \psi \\
+\frac{1}{2}\left(\Delta^{*} \overline{\psi^{\mathcal{C}}} \gamma_{5} \sigma_{2} \tau_{2} \psi+\text { H.c. }\right)-\frac{1}{4 G}\left(\sigma^{2}+\vec{\pi}^{2}+\zeta \vec{\rho}^{2}+|\Delta|^{2}\right),
\end{gathered}
$$

where $\sigma, \vec{\pi}, \vec{\rho}, \Delta$ are the collective fields with the quantum numbers of a scalar isoscalar, pseudoscalar isovector, scalar isovector, and scalar diquark, respectively.

In the mean-field approximation the collective fields are replaced with their vacuum expectation values. The presence of both baryon number and isospin chemical potentials induces a mismatch between the Fermi levels of quarks of different flavors. When the mismatch is too large, it breaks the BCS-like pairing and the system goes over to the normal phase via a first-order phase transition. Around this transition, a narrow window may exist where pairing with nonzero total momentum occurs. Such kind of pairing, well known in condensed matter physics, was first considered in the context of dense quark matter in 23], see also 24] for a comprehensive review.

In literature on color superconductivity of dense (three-color) quark matter (see 25] for recent reviews), there are in fact several other candidates for the state of dense matter in presence of a Fermi surface mismatch that compete with the inhomogeneous (FF) pairing. These include phases with a gluon condensate [26], meson condensate [27], or a meson supercurrent [28]. Let 
us explain briefly why we do not consider these possibilities in the present context. First, in a two-flavor (threecolor) color superconductor, the single plane wave FF state is equivalent to the BCS state with a uniform gluon condensate; they are connected by a gauge transformation [26]. However, this is not the case in two-color QCD where the diquark pairs are color singlets. For the same reason, the gluon condensate will be disfavored since it induces nonzero color charge (which in three-color QCD compensates for the charge of the Cooper pairs).

The possibility of a meson condensate strongly depends on the meson spectrum, and hence on the pattern of global symmetry breaking. In three-color QCD meson condensation is likely to occur in three-flavor matter where there is an octet of light pseudoscalar mesons [27]. On the other hand, in two-color QCD with two flavors, considered in this paper, the only mesons present in the low-energy spectrum of the diquark condensation phase are the pions. It can be shown that at $\mu_{I}=0$, the pion mass is equal to $\mu_{B}$ [11]. This eventually does lead to pion condensation, but only when $\mu_{I}>\mu_{B}$. There is no meson to condense inside the diquark condensation phase, thus leaving the FF pairing the only candidate for resolving the Fermi surface mismatch.

In the present paper, we will therefore take into account the possibility of inhomogeneous diquark and pion condensates. To that end, note that the Lagrangian of two-color QCD (1) is invariant (among others) under the discrete symmetry of charge conjugation of $d$-quarks. The reason why we can charge conjugate one component of the quark flavor doublet and still keep the Lagrangian gauge invariant is once again the (pseudo)reality of the doublet representation of the gauge-SU(2). This discrete symmetry remains intact even in presence of the baryon number and isospin chemical potentials, provided they are interchanged simultaneously with the charge conjugation. As a consequence, the phase diagram of two-color QCD in the $\left(\mu_{B}, \mu_{I}\right)$ plane is symmetric with respect to interchanging of the axes accompanied by the simultaneous interchanging of the diquark and pion condensates [29]. Moreover, the diquark and pion condensates never occur simultaneously, the former being favored for $\mu_{B}>\mu_{I}$, while the latter for $\mu_{B}<\mu_{I}$. This is rather obvious at high chemical potentials where quarks form a Fermi sea: at $\mu_{B}>\mu_{I}$ a Fermi sea of $d$-quarks exists, thus making formation of $u d$ pairs, and hence diquark condensation, possible. Likewise, at $\mu_{B}<\mu_{I}, \bar{d}$-quarks form a Fermi sea, leading to $u \bar{d}$ Cooper pairs and pion condensation. At low chemical potentials, the mutual exclusion of the pion and diquark condensates may be verified using chiral perturbation theory.

With the above argument in mind, we will set $\vec{\pi}=\overrightarrow{0}$ and consider the region $0<\mu_{I}<\mu_{B}$. The full phase diagram is easily constructed by reflection with respect to the line $\mu_{B}=\mu_{I}$, accompanied by the replacement of the diquark condensate with the pion one. For the coordinate dependence of the diquark condensate we will assume the simplest, single plane wave form, $\Delta e^{2 i \boldsymbol{q} \cdot \boldsymbol{x}}$ with real $\Delta$. This is the FF pairing [30]. As a side remark let us add that the FF pairing is not really the energetically most favored state. The energy may be further decreased by forming a more complicated spatial structure such as the truly inhomogeneous standing wave-like Larkin-Ovchinnikov state 31], or even various crystalline structures [32]. When the periodic structure of the order parameter is not limited to a linear combination of a finite number of plane waves, the first order transition between the BCS pairing and the FF phase is weakened and eventually becomes second order, enlarging the region in the phase diagram occupied by the inhomogeneous phase 33]. FF pairing in the context of pion condensation was investigated before in [34].

All in all, we take into account the following mean fields: $\sigma, \rho$ (standing for the third component of $\vec{\rho}$ ), and $\Delta e^{2 i \boldsymbol{q} \cdot \boldsymbol{x}}$. Note that $\rho$ gives rise to splitting of the constituent quark masses, being $M_{u, d}=m_{0}+\sigma \pm \zeta \rho$. The coordinate dependence of the diquark condensate may be removed by a unitary transformation at the price of modifying the kinetic term of quarks. After integrating out the quarks and performing the Matsubara summation, the thermodynamic potential density of the model becomes

$$
\begin{aligned}
\frac{\Omega(\sigma, \rho, \Delta, q)}{V}= & \frac{1}{4 G}\left(\sigma^{2}+\zeta \rho^{2}+\Delta^{2}\right) \\
& -T \int \frac{d^{3} \boldsymbol{k}}{(2 \pi)^{3}} \operatorname{Tr} \log \left(1+e^{-\beta \mathscr{H}_{\boldsymbol{k}}}\right) .
\end{aligned}
$$

Here $\mathscr{H}_{\boldsymbol{k}}$ is the $16 \times 16$ matrix Hamiltonian whose block elements in the Nambu space $\left(\psi_{r}, \sigma_{2} \psi_{g}^{\mathcal{C}}\right)$, read

$$
\begin{aligned}
& \left(\mathscr{H}_{\boldsymbol{k}}\right)_{11}=\boldsymbol{\alpha} \cdot(\boldsymbol{k}+\boldsymbol{q})+\gamma_{0}\left(M+\zeta \rho \tau_{3}\right)-\frac{1}{2}\left(\mu_{B}+\tau_{3} \mu_{I}\right), \\
& \left(\mathscr{H}_{\boldsymbol{k}}\right)_{12}=-\gamma_{0} \gamma_{5} \Delta \\
& \left(\mathscr{H}_{\boldsymbol{k}}\right)_{21}=+\gamma_{0} \gamma_{5} \Delta \\
& \left(\mathscr{H}_{\boldsymbol{k}}\right)_{22}=\boldsymbol{\alpha} \cdot(\boldsymbol{k}-\boldsymbol{q})+\gamma_{0}\left(M-\zeta \rho \tau_{3}\right)+\frac{1}{2}\left(\mu_{B}-\tau_{3} \mu_{I}\right),
\end{aligned}
$$

where we introduced the notation $M=m_{0}+\sigma$ for the average constituent quark mass, and $\boldsymbol{\alpha}=\gamma_{0} \gamma$ are the usual Dirac matrices. Note that the zero-point energy contribution is missing in Eq. (6) since $\operatorname{Tr} \mathscr{H}_{\boldsymbol{k}}=0$. The eigenvalues of the Hamiltonian, representing the energies of fermionic quasiparticles, cannot be found analytically. When calculating the thermodynamic potential, one has to resort to numerical diagonalization of the Hamiltonian. In [13] this technical difficulty was avoided by using an approximate analytic expression for the quasiparticle dispersion relations. In the present paper the thermodynamic potential is evaluated without introducing any further approximation.

The naive expression for the thermodynamic potential (6) is actually ill-defined because the momentum integral is badly divergent. The integral will be regulated with a three-momentum cutoff, $\Lambda$, as usual. However, with nonzero FF momentum $\boldsymbol{q}$ one must be very careful at 
what stage of the calculation the cutoff is applied. Trying to impose it naively to Eq. (6) leads to unphysical artifacts including a $\boldsymbol{q}$-dependent quadratic divergence. This was previously removed by an ad hoc subtraction [13, 35], making sure that the thermodynamic potential is independent of $\boldsymbol{q}$ once $\Delta=0$, which follows from the very definition of the FF order parameter. We would like to argue here that such a subtraction follows naturally when the thermodynamic potential is first properly regularized, and only after then the unitary transformation leading to Eq. (6) is performed. Since the argument is rather general and applies in particular also to the analogous nonrelativistic problem, we present it separately in the Appendix. The final expression for the subtracted thermodynamic potential, using the function $\Omega(\sigma, \rho, \Delta, q)$ defined by Eq. (6) , is

$$
\begin{aligned}
\Omega_{\mathrm{sub}}(\sigma, \rho, \Delta, q)= & \Omega(\sigma, \rho, \Delta, q) \\
& -\Omega(\sigma, \rho, 0, q)+\Omega(\sigma, \rho, 0,0) .
\end{aligned}
$$

The addition and subtraction operations are understood to be performed inside the integration. This prescription, differing slightly from that used in 13], also ensures that the thermodynamic potential is independent of $\boldsymbol{q}$ once $\Delta=0$. Moreover, the thermodynamic potential $\Omega_{\text {sub }}$ has a well defined minimum which determines the equilibrium state.

\section{PHASE DIAGRAM AT NONZERO BARYON AND ISOSPIN DENSITY}

The phase diagram of two-color QCD with two quark flavors in the $\left(\mu_{B}, \mu_{I}\right)$ plane and at zero temperature was first drawn in [5]. At low chemical potential its structure can be determined in a model-independent manner using the chiral perturbation theory. One thus finds that when both chemical potentials are smaller than $m_{\pi}$, the vacuum mass of the pion-diquark multiplet of pseudo-NG bosons of the spontaneously broken $\mathrm{SU}(4)$ flavor symmetry, the system is in the vacuum phase. Only the chiral condensate is nonzero, equal to its vacuum value independently of the chemical potential. As soon as one of the chemical potentials, say $\mu_{B}$ (remember the reflection symmetry of the phase diagram), exceeds $m_{\pi}$, the mode carrying the associated charge - in this case the diquark - condenses via a second-order phase transition. Since this picture relies just on the symmetry and the pattern of its spontaneous breaking, it is not surprising that results obtained within chiral perturbation theory, lattice simulations [15], and model calculations [11] are in good quantitative agreement. At moderate chemical potentials one therefore finds BEC phases with a diquark (for $\mu_{B}>\mu_{I}$ ) or pion condensate (for $\mu_{B}<\mu_{I}$ ), separated by a first-order phase transition line at $\mu_{B}=\mu_{I}$.

On the other hand, at high chemical potential one expects the physics of the system to be dominated by the Fermi sea of quarks. Spontaneous symmetry breaking is realized by a formation of Cooper pairs via the standard BCS scenario. Since the Cooper pairs carry the same quantum numbers as the condensing modes in the low-density BEC phase, there is no phase transition between the two regimes despite the fact that they feature qualitatively different physical behavior. One speaks of the BCS-BEC crossover 36. In the context of two-color QCD, this was studied in 12]; we will comment on it later in Sections $\mathrm{V}$ and VI.

The qualitative shape of the phase diagram at high chemical potential was conjectured in [5] based on the experience with real, three-color QCD at weak coupling. The essence of the argument is as follows. Close to the $\mu_{B}$ and $\mu_{I}$ axes, one will find diquark and pion condensates as predicted by BCS theory. However, since both of them involve pairing of quarks of different flavors, they will become less and less favored towards the "diagonal" of the phase diagram; $\mu_{I}$ generates a mismatch between the Fermi levels of $u$ and $d$ quarks in the diquark condensation phase, while $\mu_{B}$ plays the same role in the pion condensation phase. When the mismatch becomes large enough, the BCS pairing is no longer energetically favorable and it breaks in a first-order phase transition. In a narrow window around this transition, a phase where Cooper pairs carry nonzero total momentum may exist. Even after the cross-flavor pairing is made impossible, quarks of the same flavor can still pair, since there is no Fermi level mismatch by definition. Such pairs are required to carry nonzero spin or orbital momentum by the Pauli principle [10]. In a narrow strip along the diagonal of the phase diagram, the chemical potential of $d$-quarks is almost zero, and they should therefore be confined and exhibit the one-flavor chiral condensate. Thus, only the $u$-quarks will pair in this region.

We employed the NJL model to verify the conjecture made in [5]. In order to obtain concrete numbers, we used the two-color NJL parameter set, established in 20]: $G=$ $7.23 \mathrm{GeV}^{-2}, m_{0}=5.4 \mathrm{MeV}$, and $\Lambda=657 \mathrm{MeV}$. This corresponds to the physical pion mass $m_{\pi}=140 \mathrm{MeV}$, pion decay constant $f_{\pi}=75.4 \mathrm{MeV}$, and (one-flavor) chiral condensate $\left\langle\bar{\psi}_{u} \psi_{u}\right\rangle=(-218 \mathrm{MeV})^{3}$. The latter two quantities were obtained from usual three-color values by rescaling with respect to the number of colors. The parameter $\zeta$ is treated as free in this paper.

While we included the FF-type diquark and pion condensates as well as independent chiral condensates for $u$ and $d$ quarks, we did not, for the sake of simplicity, take into account spin-one pairing. This would most likely further split the region in the upper right corner of the phase diagram into domains with or without spin-one pairing of individual flavors. On the other hand, it is not expected to alter the structure that we find. The reason is that spin-one pairing is usually characterized by a small pairing gap as compared to spin-zero pairing, and the pairing moreover does not occur isotropically on the whole Fermi surface. Consequently, the energy gain from spin-one pairing is minute so that it cannot compete with phases with other types of pairing. 


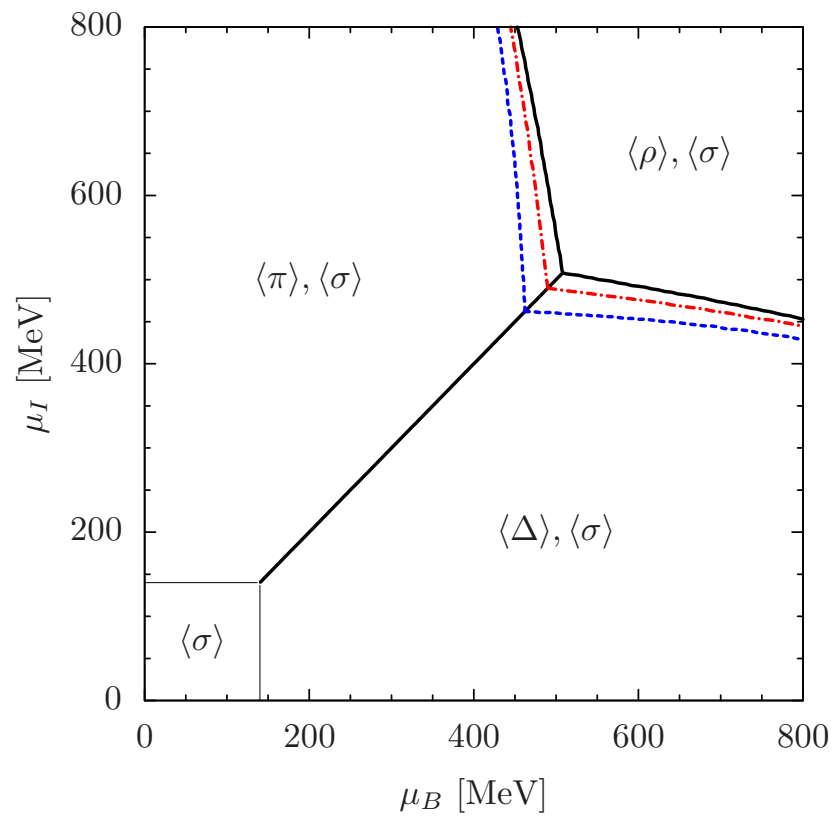

FIG. 1. Phase diagram of two-color QCD with two quark flavors in the $\left(\mu_{B}, \mu_{I}\right)$ plane at zero temperature. Various phases are labeled by the corresponding nonzero condensates. The $\rho$ condensate in the upper right corner only appears at $\zeta \neq 0$. Thick and thin lines denote first- and second-order transitions, respectively. The black solid lines were obtained with $\zeta=0(\alpha=0.5)$, the red dash-dotted lines with $\zeta=0.8$ $(\alpha=0.1)$, and the blue dashed lines with $\zeta=1(\alpha=0)$. The position of the transitions at high chemical potentials only changes appreciably for $\zeta$ close to one. The $\zeta=0.6$ lines would almost coincide with the $\zeta=0$ ones shown.

The phase diagram at zero temperature obtained with the above set of parameters and condensates taken into account is shown in Fig. 11. One can see that at moderate chemical potentials, there is no FF phase and the system goes directly to the phase with just chiral condensate(s). We avoid calling this a normal phase, because the size of the chiral condensate(s) depends sensitively on the parameter $\zeta$. For $\zeta=0, \rho=0$ and the common chiral condensate of the $u$ and $d$ quarks is indeed very small. As $\zeta$ increases, the flavor chiral condensates $\sigma_{u, d}=\sigma \pm \zeta \rho$ split, or in other words, $\rho$ becomes nonzero. Naturally, $\sigma_{u}$ remains very small since the $u$-quark chemical potential is very large, while $\sigma_{d}$ grows towards the diagonal of the phase diagram. In fact, in the $\mathrm{U}(4)$ symmetric limit $(\zeta=1)$ the mean-field gap equations for $\sigma_{u}$ and $\sigma_{d}$ in absence of diquark and pion condensates completely decouple. The $d$-quark condensate $\sigma_{d}$ is then at the diagonal of the phase diagram (and around) equal to its vacuum value. As soon as the $\mathrm{U}(1)_{\mathrm{A}}$ symmetry is explicitly broken, the flavors become entangled and the $d$ quark condensate drops fast with increasing $\alpha$. However, despite the strong dependence of the flavor chiral condensates on $\zeta$, the position of the phase transition does not change much. Some shift is actually only visible as $\zeta$ ap-

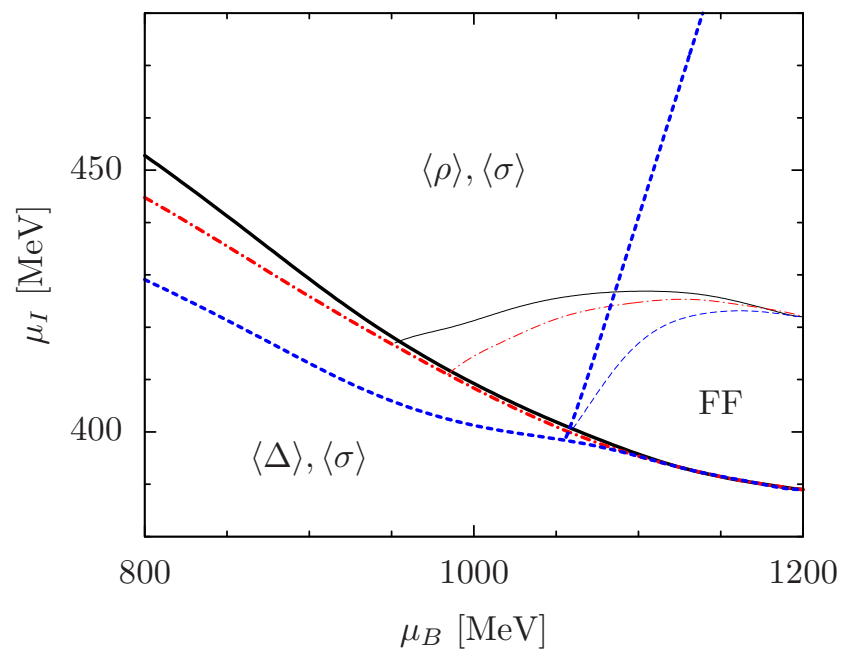

FIG. 2. The high chemical potential corner of the phase diagram. The notation for the lines is the same as in Fig. 1 The region where the momentum $\boldsymbol{q}$ is nonzero is indicated as "FF". The additional thick blue dashed line is associated with the first-order chiral restoration transition at $\xi=1$, explained in the text. Note that the chemical potential used to label the horizontal axis is the baryon number one so that the corresponding quark chemical potential in the range displayed is still below the cutoff $\Lambda$. Hence, cutoff artifacts, though nonnegligible, will not alter the qualitative structure of the phase diagram.

proaches one. Note that for the sake of clarity, we do not indicate the BCS-BEC crossover in Fig. 1. This appears at $\mu_{B, I} \approx 229 \mathrm{MeV}$; its position is to a very good precision determined by the expression $\left(2 M_{0} m_{\pi}^{2}\right)^{1 / 3}$, where $M_{0}$ is the constituent quark mass in the vacuum [12].

The domain of high chemical potentials is shown in detail in Fig. 2, The FF phase appears only at rather high chemical potentials. This is in agreement with the observation that at in strongly coupled systems the region with the FF phase is diminished [13, 37]. In quark matter one cannot tune the coupling arbitrarily. Nevertheless, the weakly coupled BCS pairing is achieved effectively at high chemical potential since the ratio $\Delta / \mu_{B}$ drops with increasing chemical potential. Even though we used a different parameter set than in [13] and a more refined method to evaluate the thermodynamic potential at nonzero FF momentum $\boldsymbol{q}$, our results are in reasonable quantitative agreement.

As $\zeta$ increases, the FF phase is obviously expelled from the phase diagram. This is a direct consequence of the fact that the energy gain from FF pairing is rather small due to both the small size of the pairing gap and the fact that pairing occurs only on a small part of the Fermi surface. It therefore becomes energetically more favorable to form a state with a large splitting of constituent $u$ and $d$ quark masses, which in turn inhibits the crossflavor pairing. On the other hand, one should note that in the FF phase, $\rho$ does take a nonzero, albeit small 


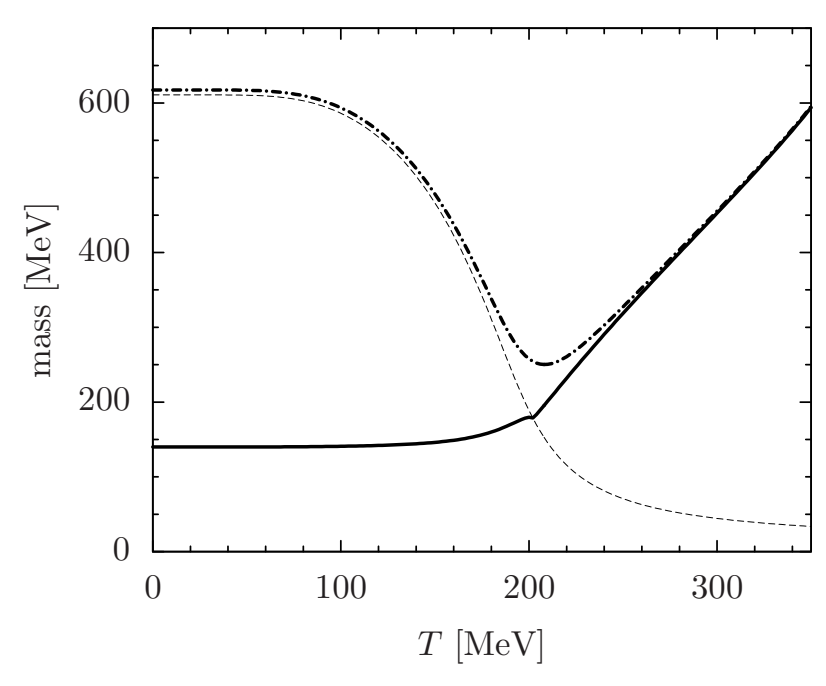

FIG. 3. Meson masses as a function of temperature at zero chemical potential. Solid line: common mass of pions and the (anti)diquark. Dash-dotted line: sigma mass. The thin dashed line indicates the position of the two-particle continuum, that is, twice the constituent quark mass.

value. The reason is that the difference of the constituent quark masses partially compensates for the chemical potential mismatch, thus reducing the mismatch of Fermi momenta.

Fig. 2 also features an additional first-order transition line which occurs only for $\zeta=1$ and is defined by the condition $\mu_{B}-\mu_{I}=$ const. This can be explained using the observation made above that at $\zeta=1$ the gap equations for $\sigma_{u}$ and $\sigma_{d}$ completely decouple. The two flavors then live their separate lives, and instead of $\mu_{B, I}$ it would be more convenient to use the basis $\mu_{u, d}=\frac{1}{2}\left(\mu_{B} \pm \mu_{I}\right)$. The first-order line in question corresponds to the critical value of $\mu_{d}$ at which a strong first-order chiral symmetry breaking/restoration phase transition occurs. The critical value, $\mu_{d} \approx 330 \mathrm{MeV}$, extracted from the phase diagram in Fig. 2, is in excellent agreement with an elementary calculation within a one-flavor version of our model (5), keeping the chiral condensate $\sigma_{d}$ as the sole order parameter.

Once $\zeta$ drops below one, that is, axial $\mathrm{U}(1)_{\mathrm{A}}$ symmetry is explicitly broken by nonzero $\alpha$, the two quark flavors become entangled. This smears the phase transition in the $d$-quark sector, making it a smooth crossover. We have checked numerically that there is no sharp phase transition already for very small $\alpha$, and for the case $\alpha=0.1$ displayed in Fig. 2 by red dash-dotted lines, the crossover is already rather gradual.

\section{COLLECTIVE MODES}

With the semi-bosonized Lagrangian (5), the propagators of the bosonic collective modes are straightforwardly derived by integrating out the quarks and taking the second variational derivative with respect to the collective fields $\chi_{i}$. The general expression for the inverse propagator at imaginary (Matsubara) frequency $i \Omega_{N}$ is [20]

$$
\begin{aligned}
& D_{i j}^{-1}\left(i \Omega_{N}, \boldsymbol{p}\right)=\frac{1}{2 G} \delta_{i j}+T \sum_{n} \int \frac{d^{3} \boldsymbol{k}}{(2 \pi)^{3}} \\
& \times \operatorname{Tr}\left[\frac{\partial \mathscr{H}}{\partial \chi_{i}} \frac{1}{i\left(\omega_{n}+\Omega_{N}\right)-\mathscr{H}_{\boldsymbol{k}+\frac{p}{2}}} \frac{\partial \mathscr{H}}{\partial \chi_{j}} \frac{1}{i \omega_{n}-\mathscr{H}_{\boldsymbol{k}-\frac{p}{2}}}\right] .
\end{aligned}
$$

This formula is valid for the $\sigma, \vec{\pi}, \Delta$ modes we are interested in. For modes from the other chiral multiplet, a factor $\zeta$ must be added to the constant part of the propagator. In general, when some of the condensates break the symmetry spontaneously, different modes may mix. We will only give explicit expressions for the propagators in the case that just the isospin-singlet chiral condensate $(\sigma)$ is nonzero. This covers the connected domain in the phase diagram containing the origin. Since the BEC transition to either diquark or pion condensed phase is of second order, the knowledge of these propagators allows one to locate these transitions by means of the Thouless criterion [38]. For the sake of simplicity of the resulting formulas, we will set the external three-momentum to zero. (A more complete analysis of collective modes may be found in [20]; the evolution of the collective mode spectrum as a function of baryon chemical potential has also been studied on the lattice [39].) With these simplifications in mind, the inverse propagators of the six modes of interest to us read, after analytic continuation to general complex frequency $\omega$, 


$$
\begin{aligned}
& D_{\sigma}^{-1}(\omega)=\frac{1}{2 G}-4 \sum_{e= \pm} \int \frac{d^{3} \boldsymbol{k}}{(2 \pi)^{3}} \frac{\boldsymbol{k}^{2}}{\epsilon_{\boldsymbol{k}}^{2}}\left[\frac{1-f\left(e \xi_{\boldsymbol{k}}^{e}\right)-f\left(e \xi_{\boldsymbol{k}}^{-e}\right)}{\omega+2 e \epsilon_{\boldsymbol{k}}}+\frac{1-f\left(e \hat{\xi}_{\boldsymbol{k}}^{e}\right)-f\left(e \hat{\xi}_{\boldsymbol{k}}^{-e}\right)}{\omega+2 e \epsilon_{\boldsymbol{k}}}\right], \\
& D_{\pi^{0}}^{-1}(\omega)=\frac{1}{2 G}-4 \sum_{e= \pm} \int \frac{d^{3} \boldsymbol{k}}{(2 \pi)^{3}}\left[\frac{1-f\left(e \xi_{\boldsymbol{k}}^{e}\right)-f\left(e \xi_{\boldsymbol{k}}^{-e}\right)}{\omega+2 e \epsilon_{\boldsymbol{k}}}+\frac{1-f\left(e \hat{\xi}_{\boldsymbol{k}}^{e}\right)-f\left(e \hat{\xi}_{\boldsymbol{k}}^{-e}\right)}{\omega+2 e \epsilon_{\boldsymbol{k}}}\right], \\
& D_{\pi^{+}}^{-1}(\omega)=\frac{1}{2 G}-8 \sum_{e= \pm} \int \frac{d^{3} \boldsymbol{k}}{(2 \pi)^{3}} \frac{1-f\left(e \xi_{\boldsymbol{k}}^{e}\right)-f\left(e \hat{\xi}_{\boldsymbol{k}}^{-e}\right)}{\omega+\mu_{I}+2 e \epsilon_{\boldsymbol{k}}} \\
& D_{\Delta}^{-1}(\omega)=\frac{1}{2 G}-8 \sum_{e= \pm} \int \frac{d^{3} \boldsymbol{k}}{(2 \pi)^{3}} \frac{1-f\left(e \xi_{\boldsymbol{k}}^{e}\right)-f\left(e \hat{\xi}_{\boldsymbol{k}}^{e}\right)}{\omega+\mu_{B}+2 e \epsilon_{\boldsymbol{k}}} .
\end{aligned}
$$

We use the usual notation for the Lorentz-invariant dispersion relation, $\epsilon_{\boldsymbol{k}}=\sqrt{\boldsymbol{k}^{2}+M^{2}}$, and the Fermi-Dirac distribution function, $f(x)=1 /\left(e^{\beta x}+1\right)$. Furthermore, $\xi_{\boldsymbol{k}}^{e}=\epsilon_{\boldsymbol{k}}+\frac{e}{2}\left(\mu_{B}+\mu_{I}\right)$ denotes the in-medium energy of the $u$-(anti)quark, and $\hat{\xi}_{\boldsymbol{k}}^{e}=\epsilon_{\boldsymbol{k}}+\frac{e}{2}\left(\mu_{B}-\mu_{I}\right)$ that of the $d$-(anti)quark. The propagators of $\pi^{-}$and of the antidiquark are obtained from those of $\pi^{+}$and the diquark by changing the sign of $\mu_{I}$ and $\mu_{B}$, respectively.

In Fig. 3 we plot the meson masses as a function of temperature at zero chemical potential. Note that thanks to the unbroken $\mathrm{SO}(5)$ symmetry, the pions and diquarks are exactly degenerate even away from the chiral limit. The masses are determined from the zeroes of the real part of the inverse propagators (8). As long as the mode is stable, this definition coincides with the physical, pole mass. Once the two-particle threshold is crossed, the definition of the mass becomes ambiguous and it is more convenient to discuss the full spectral density, $\rho(\omega)$. This is obtained from the imaginary part of the retarded Green's function, and is related to the inverse propagators (8) by

$$
\rho(\omega)=-\frac{\operatorname{Im} D^{-1}(\omega+i \varepsilon)}{\left[\operatorname{Re} D^{-1}(\omega+i \varepsilon)\right]^{2}+\left[\operatorname{Im} D^{-1}(\omega+i \varepsilon)\right]^{2}} .
$$

The spectral densities as a function of frequency for five different values of temperature $(140,180,220,260,300 \mathrm{MeV})$ are plotted in Fig. 4. One can see clearly that all modes constitute well-defined quasiparticles below the chiral restoration crossover; the pions and diquarks are bound and therefore stable, while the sigma mass is just above the threshold and thus gives rise to a very sharp peak in the spectrum. Once the temperature increases, the constituent quark mass rapidly drops, the level of the two-particle continuum consequently decreases, and all mesons become broad and eventually more or less washed out of the spectrum. For temperatures above about $300 \mathrm{MeV}$ the notion of mass becomes meaningless.

The range of stability of the modes is more precisely determined by the so-called dissociation temperature, defined as the temperature at which the quasiparticle pole in the propagator (8) enters the two-particle continuum [40]. (Note that a different definition of the dissociation temperature was used in [12].) In Fig. 5 the phase diagram at zero isospin chemical potential is drawn with

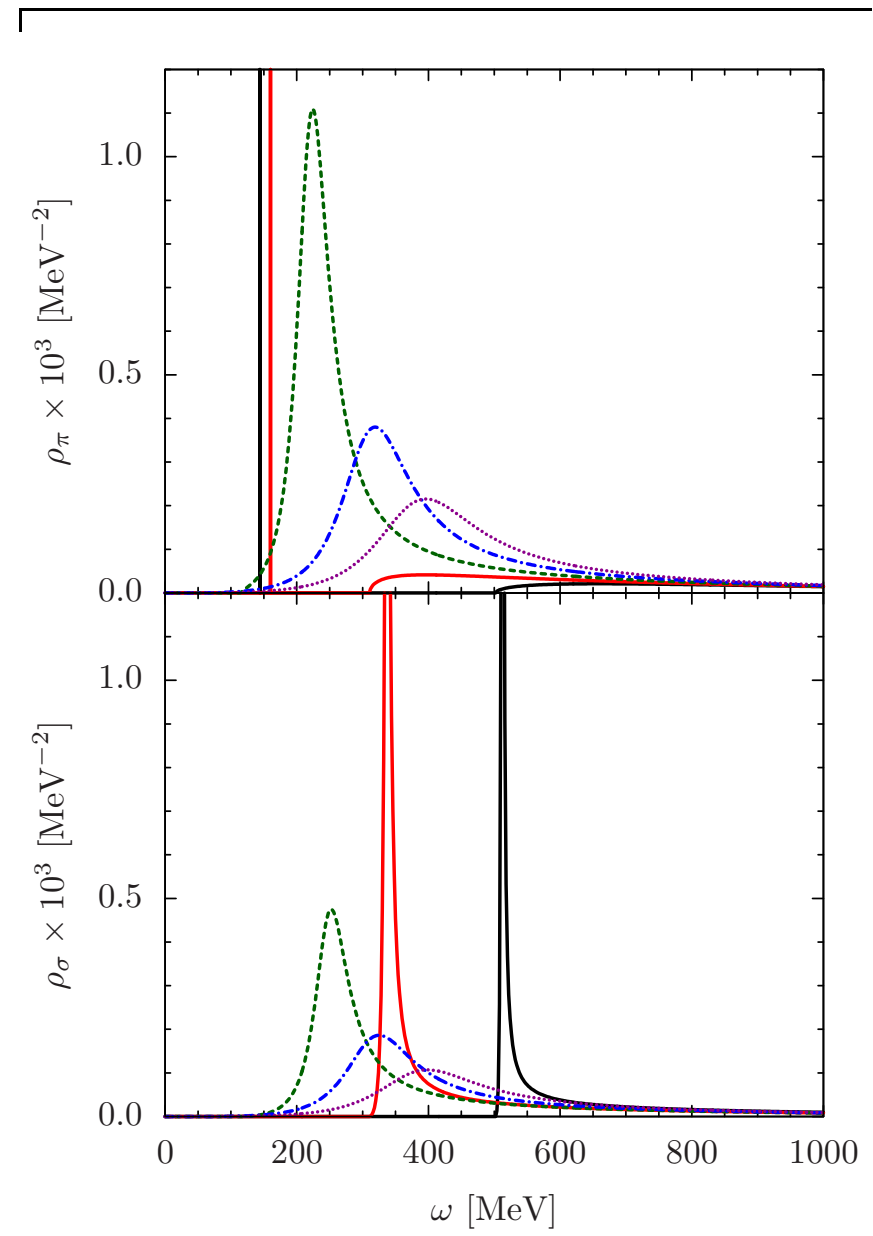

FIG. 4. Spectral densities of the pions and diquarks (upper panel) and sigma (lower panel) as a function of frequency at zero chemical potential. The various lines correspond to temperature of: $140 \mathrm{MeV}$ (black, solid), $180 \mathrm{MeV}$ (red, solid), $220 \mathrm{MeV}$ (green, dashed), $260 \mathrm{MeV}$ (blue, dashdotted), $300 \mathrm{MeV}$ (magenta, dotted).

the dissociation temperatures of the pions, the diquark and antidiquark indicated. We can see that our definition of the dissociation temperature nicely coincides with the position of the BCS-BEC crossover, to be discussed in more detail in the following section. It is suitable to re- 


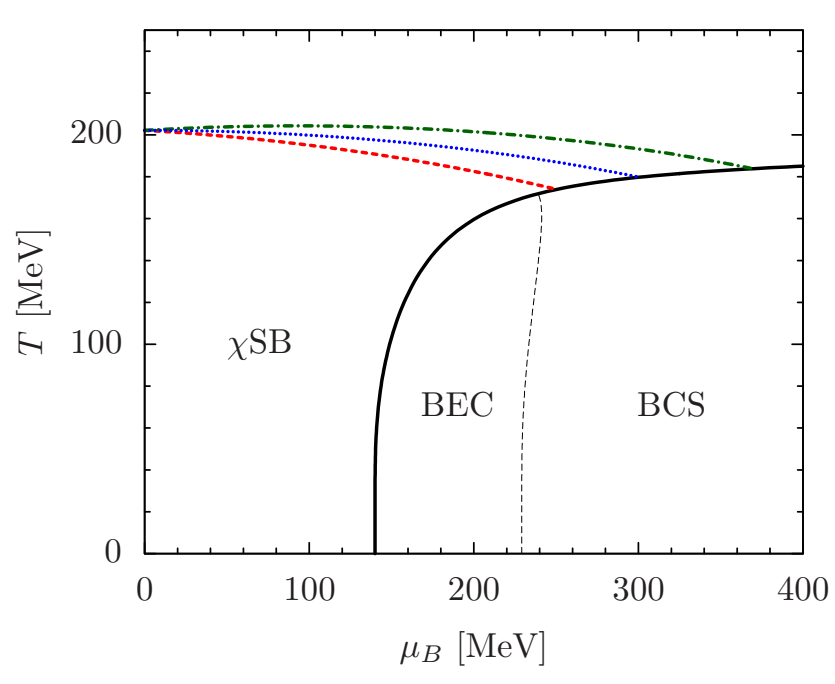

FIG. 5. Phase diagram of two-color QCD at zero isospin chemical potential. The thick black solid line denotes the second-order diquark condensation transition. The thin black dashed line indicates the position of the BCS-BEC crossover, defined by the condition $M=\mu_{B} / 2$. The dissociation temperatures of the collective modes are represented by the: red dashed line for the diquark, green dash-dotted line for the antidiquark, blue dotted line for the pions. The region where the physics is dominated by the chiral condensate is labeled as $\chi \mathrm{SB}$.

mark here that when the effects of confinement are taken into account, for example by coupling the NJL model to the Polyakov loop, the transition temperature becomes somewhat higher than in Fig. 5 [20. However, the qualitative structure of the phase diagram is not changed. Also, it should be stressed that adding the Polyakov loop would only modify the results of this section. It would have no effect at all on the phase diagram in the $\left(\mu_{B}, \mu_{I}\right)$ plane at zero temperature, discussed in Sec. IV, and it plays an absolutely negligible role in the thermodynamics of diquark BEC, to be investigated in Sec. VI [16].

\section{THERMODYNAMICS OF DIQUARK BOSE-EINSTEIN CONDENSATION}

The main virtue of two-color QCD, providing a strong motivation for its study, is the possibility of lattice Monte Carlo simulations. It is therefore of utmost importance to try to match model calculations to available lattice data. To this end, one should note that in order to have a positive determinant of the Dirac operator that in turn makes the importance sampling possible, one needs an even number of quark flavors with the same chemical potentials. The phase diagram in the $\left(\mu_{B}, \mu_{I}\right)$ plane investigated in Sec. IV is therefore not accessible to current lattice techniques. The lattice literature on twocolor QCD at nonzero density invariably addresses the case of zero isospin chemical potential, where the indi-

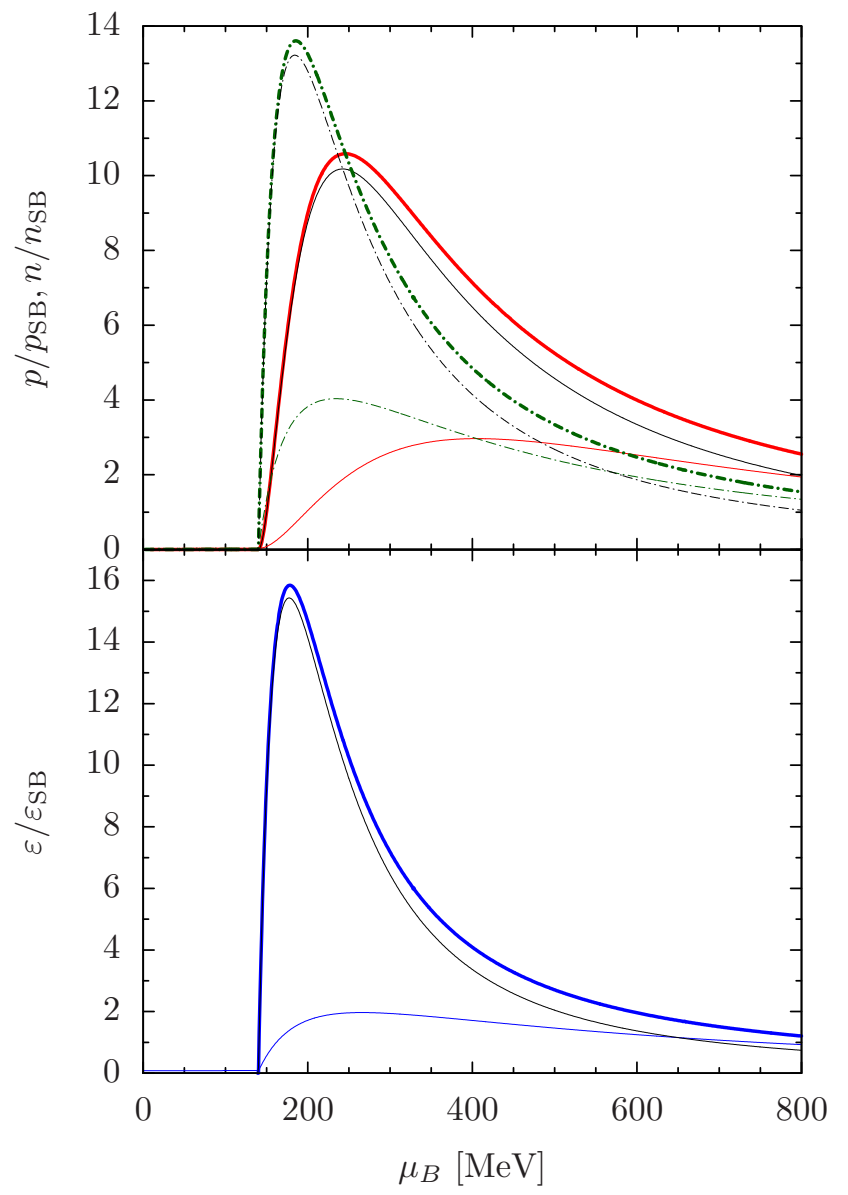

FIG. 6. Pressure and baryon density (upper panel) and energy density (lower panel), normalized to the Stefan-Boltzmann values, at zero isospin chemical potential as a function of baryon number chemical potential. The thick lines correspond to $T=0 \mathrm{MeV}$ with the following notation: the red solid line for pressure, the green dash-dotted line for baryon density, and the blue solid line for energy density. The thin lines with the same line notation show the results for $T=50 \mathrm{MeV}$. The thin black lines, almost coinciding with the zero-temperature NJL values, are the results of chiral perturbation theory at leading order.

vidual quark flavors indeed share the common chemical potential, $\mu_{B}$. The latest simulations [16] study the thermodynamic properties of two-color QCD with two quark flavors at low temperature as a function of the baryon chemical potential. (They also calculate the gluon propagator which, however, cannot be determined in model approaches based on the flavor symmetry.)

Within our NJL model, we calculate the pressure from the thermodynamic potential (7), making an appropriate constant subtraction to ensure that the vacuum pressure is zero. The baryon number density and energy density are in turn obtained from the pressure using the thermodynamic identities, $n_{B}=\partial p / \partial \mu_{B}$ and $\varepsilon=-p+\mu_{B} n_{B}+T s$, where the entropy density reads $s=\partial p / \partial T$. Since at high chemical potentials the system 
is expected to behave as a weakly interacting Fermi gas, it is convenient to normalize these thermodynamic quantities by the Stefan-Boltzmann (SB) values, corresponding to a free massless Fermi gas. Taking into account that two-color QCD with two quark flavors includes four different species of Dirac fermions, these read

$p_{\mathrm{SB}}=\frac{\mu_{B}^{4}}{48 \pi^{2}}+\frac{\mu_{B}^{2} T^{2}}{6}+\frac{7 \pi^{2} T^{4}}{45}, \quad n_{\mathrm{SB}}=\frac{\mu_{B}^{3}}{12 \pi^{2}}+\frac{\mu_{B} T^{2}}{3}$,

and $\varepsilon_{\mathrm{SB}}=3 p_{\mathrm{SB}}$. The numerical results for two different temperatures are shown by the colored lines in Fig. 6 [41]. Remarkably, even for the highest chemical potentials displayed, the pressure and density are still rather far from the SB limit. In the mean-field approximation, this is obviously due to the pairing gap.

Since near the diquark BEC phase transition, the theory behaves like a dilute Bose gas rather than a system of noninteracting quasiparticles, described by the NJL model in the mean-field approximation, we calculate for comparison the same quantities in a model with elementary scalar degrees of freedom. For the sake of simplicity, we use the $\mathrm{O}(6)$ linear sigma model at tree level (corresponding to zero temperature). This is defined by the Euclidean Lagrangian

$$
\mathscr{L}_{\mathrm{l} \sigma \mathrm{m}}=\frac{1}{2}\left(\partial_{\mu} \vec{\phi}\right)^{2}-\frac{1}{2} M^{2} \vec{\phi}^{2}+\frac{1}{4} \lambda\left(\vec{\phi}^{2}\right)^{2}-H \sigma,
$$

$\vec{\phi}=(\sigma, \vec{\pi}, \operatorname{Re} \Delta, \operatorname{Im} \Delta)^{T}$, where $\vec{\pi}$ is the pion triplet and $\Delta$ the complex diquark field. The parameter $H$ breaks the $\mathrm{O}(6)$ symmetry explicitly down to $\mathrm{O}(5)$. The baryon chemical potential $\mu_{B}$ is incorporated by the replacement of the ordinary derivatives with covariant ones, acting nontrivially only on the diquark, $D_{0} \Delta=\left(\partial_{0}-\mu_{B}\right) \Delta$, and $D_{0} \Delta^{\dagger}=\left(\partial_{0}+\mu_{B}\right) \Delta^{\dagger}$.

The tree-level analysis of this model is straightforward, so we proceed directly to the results. The negative mass squared triggers condensation of the field, which, in the vacuum, is aligned with the explicit symmetry breaking term, $\sigma \equiv f_{\pi}=\sqrt{\left(M^{2}+m_{\pi}^{2}\right) / \lambda}$, where the pion mass is determined implicitly by $H=f_{\pi} m_{\pi}^{2}$. As soon as the baryon chemical potential exceeds the pion mass, diquarks condense. The chiral and diquark condensates are then in general parameterized by $\sigma=v \cos \theta$ and $\Delta=v \sin \theta$. For the magnitude $v$ and the angle $\theta$ one finds the expressions

$$
v^{2}=\frac{M^{2}+\mu_{B}^{2}}{\lambda}, \quad \cos \theta=\frac{m_{\pi}^{2}}{\mu_{B}^{2}} \sqrt{\frac{M^{2}+m_{\pi}^{2}}{M^{2}+\mu_{B}^{2}}} .
$$

The unphysical Lagrangian parameter $M^{2}$ may be eliminated in favor of the mass of the "radial" excitation, conventionally identified with the sigma particle, $m_{\sigma}^{2}=$ $2 M^{2}+3 m_{\pi}^{2}$. All quantities of interest can then be expressed in terms of observables. For instance, the diquark condensate is given by

$$
\Delta=f_{\pi} \sqrt{1-\left(\frac{m_{\pi}}{\mu_{B}}\right)^{4}+2 \frac{\mu_{B}^{2}-m_{\pi}^{2}}{m_{\sigma}^{2}-m_{\pi}^{2}}}
$$

Note that the results of chiral perturbation theory [2] are recovered by taking the limit $m_{\sigma} \rightarrow \infty$. Also, the expressions for the chiral condensate, $\sigma=f_{\pi} m_{\pi}^{2} / \mu_{B}^{2}$, and the baryon density, $n_{B}=\mu_{B} \Delta^{2}$, are actually identical to those in the chiral perturbation theory. (The baryon density, nevertheless, takes on a different value due to the modified value of the diquark condensate.) It is interesting to observe that as the chemical potential approaches $m_{\pi}$ from the right, the predictions of the linear sigma model and chiral perturbation theory are related by $\Delta_{\mathrm{l} \sigma \mathrm{m}} / \Delta_{\chi \mathrm{PT}}=m_{\sigma} / \sqrt{m_{\sigma}^{2}-m_{\pi}^{2}}$. This means that as soon as the diquark condensate is nonzero, a finite sigma mass drives the results off the predictions of chiral perturbation theory even in the limit of zero density. Given the above solution of the gap equations, it is straightforward to calculate the thermodynamic quantities. Using again the normalization with respect to the SB values, one finds

$$
\begin{aligned}
& \frac{p_{\mathrm{l} \sigma \mathrm{m}}}{p_{\mathrm{SB}}}=24 \pi^{2}\left(\frac{f_{\pi}}{m_{\pi}}\right)^{2}\left(1-\frac{1}{x^{2}}\right)^{2}\left(\frac{1}{x^{2}}+\frac{1}{\tau^{2}-1}\right), \\
& \frac{n_{\mathrm{l} \sigma \mathrm{m}}}{n_{\mathrm{SB}}}=\frac{12 \pi^{2}}{x^{2}}\left(\frac{f_{\pi}}{m_{\pi}}\right)^{2}\left(1-\frac{1}{x^{4}}+2 \frac{x^{2}-1}{\tau^{2}-1}\right), \\
& \frac{\varepsilon_{\mathrm{l} \sigma \mathrm{m}}}{\varepsilon_{\mathrm{SB}}}=8 \pi^{2}\left(\frac{f_{\pi}}{m_{\pi}}\right)^{2} \frac{x^{2}-1}{x^{4}}\left(1+\frac{3}{x^{2}}+\frac{3 x^{2}+1}{\tau^{2}-1}\right),
\end{aligned}
$$

where we used the shorthand notation $x=\mu_{B} / m_{\pi}$ and $\tau=m_{\sigma} / m_{\pi}$. Similar expressions were obtained for pion condensation in [42]. It is amusing, though perhaps not very physical, that all the expressions (10) have a nonzero limit as $x \rightarrow \infty$. This takes even the same value for all three observables, $24 \pi^{2} f_{\pi}^{2} /\left(m_{\sigma}^{2}-m_{\pi}^{2}\right)$.

Taking the values of $m_{\pi}$ and $f_{\pi}$ over from the NJL model, these predictions of the linear sigma model depend on a single parameter, namely $\tau$. The parameterfree results in the limit $\tau \rightarrow \infty$ (that is, chiral perturbation theory) are shown in Fig. 6] by the thin black lines. In fact, adjusting $\tau \approx 9$, one would find that the NJL and linear sigma model curves lie exactly on top of each other up to $\mu_{B}$ about $400 \mathrm{MeV}$, and remain very close in the whole range of chemical potentials displayed in Fig. 6. A cautious reader may wonder why this is so, remembering our claim in Sec. IV that at $\mu_{B} \approx 229 \mathrm{MeV}$ a crossover from the BEC behavior to quark matter occurs. The reason for this unexpected agreement even after the crossover to quark matter, defined by the change of sign of the expression $M-\mu_{B} / 2$, is that the quark excitations will keep a large energy gap. At low temperatures, they will not be excited and the bulk thermodynamic quantities will be dominated by the condensate (and its fluctuations, as we will see later).

Let us now compare our calculation based on chiral modes with the lattice data. A glance at the results presented in Ref. [16] reveals that all three thermodynamic observables considered here have substantially different values. This is, of course, not so surprising on account of the fact that we made no attempt to fit our model 
parameters to the lattice data. However, this issue deserves more care, and we would now like to explain to what extent the lattice data actually can be explained with chiral models.

First of all, one should note that the lattice computations are always performed with a nonzero source for the diquark operator. While to obtain physical predictions one should eventually take the limit of zero source, for the sake of comparison it is technically much easier to include the nonzero source in the model calculations. Indeed, in the NJL model, adding a diquark source amounts simply to shifting the diquark field $\Delta$ in the Yukawa interaction term in (5), and consequently also in the quasifermion contribution to the thermodynamic potential (6). In the linear sigma model Lagrangian (9), we would replace $H \sigma$ with $H \sigma+J \operatorname{Re} \Delta, J$ being the diquark source. The resulting gap equations can still be solved analytically, albeit implicitly, in the chiral perturbation theory limit, that is, $\tau \rightarrow \infty$ [2]. Writing the sources $H$ and $J$ in terms of a new angle $\varphi$ as $H=f_{\pi} m_{\pi}^{2} \cos \varphi$ and $J=f_{\pi} m_{\pi}^{2} \sin \varphi$, the gap equation for $\theta$, which determines the orientation of the condensate, reads

$$
x^{2} \sin \theta \cos \theta=\sin (\theta-\varphi) .
$$

Once this equation is solved for $\theta$, the thermodynamic observables are obtained from the generalization of Eq. (10) to nonzero source in the limit $\tau \rightarrow \infty$,

$$
\begin{aligned}
\frac{p_{\chi \mathrm{PT}}}{p_{\mathrm{SB}}} & =48 \pi^{2}\left(\frac{f_{\pi}}{m_{\pi}}\right)^{2} \frac{1}{x^{4}}\left[\frac{1}{2} x^{2} \sin ^{2} \theta+\cos (\theta-\varphi)-1\right], \\
\frac{n_{\chi \mathrm{PT}}}{n_{\mathrm{SB}}} & =\frac{12 \pi^{2}}{x^{2}}\left(\frac{f_{\pi}}{m_{\pi}}\right)^{2} \sin ^{2} \theta, \\
\frac{\varepsilon_{\chi \mathrm{PT}}}{\varepsilon_{\mathrm{SB}}} & =16 \pi^{2}\left(\frac{f_{\pi}}{m_{\pi}}\right)^{2} \frac{1}{x^{4}}\left[\frac{1}{2} x^{2} \sin ^{2} \theta-\cos (\theta-\varphi)+1\right] .
\end{aligned}
$$

The results of a sample calculation using chiral perturbation theory with a nonzero diquark source are shown in Fig. 7. Since we were not able to fix the input parameters in the vacuum, we simply fit the prediction of chiral perturbation theory to lattice results at nonzero chemical potential. We thus obtain $\varphi \approx 0.10, f_{\pi} / m_{\pi} \approx 0.21$, and $m_{\pi} \approx 0.59$ in lattice units. (We used only the data for baryon density in the fit, since the pressure may get a nontrivial contribution from the gluon sector of QCD. Also, only the points with $\mu_{B} / m_{\pi} \lesssim 2$ were taken into account.) Doing this, we merely wish to demonstrate that a part of the lattice data can be explained using a model based just on the chiral symmetry. Obviously, the same numerical values can also be obtained with the NJL model; we did not do that since it would require tuning an even larger number of parameters at will.

It is clear that the high numerical values of the NJL prediction plotted in Fig. 6] do not present a problem. In fact, Eq. (10) illustrates convincingly that they scale sensitively with the ratio $f_{\pi} / m_{\pi}$. What is a problem, however, is the large ratio of the heights of the peaks in the energy density, and in the pressure and baryon density,

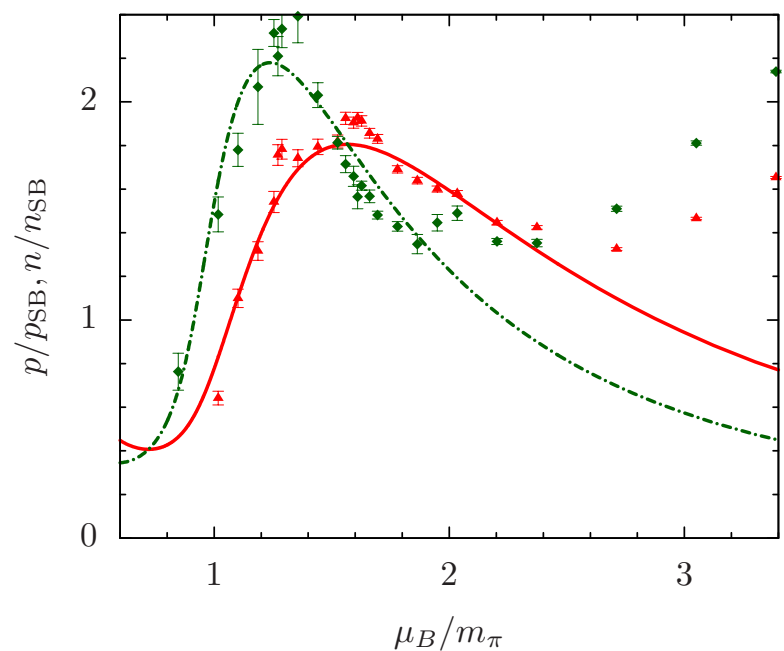

FIG. 7. Comparison of lattice results [16] (we are indebted to S. Hands for providing us with the data) and leading order chiral perturbation theory with nonzero diquark source. The line notation is the same as in Fig. 6] red solid for pressure and green dash-dotted for baryon density.

as observed on the lattice [16]. This is the reason why we did not plot the energy density in Fig. 7 at all. Both the mean-field NJL model and the linear sigma model at tree level (and in turn the chiral perturbation theory at the leading order) predict all three peaks to have heights of roughly the same magnitude. Even though we cannot offer and ultimate resolution of this discrepancy, we propose a possible explanation.

Our reasoning goes back to the thermodynamic identity $\varepsilon=-p+\mu_{B} n_{B}+T s$. Note that the lattice simulations were performed at low temperature, roughly an order of magnitude smaller than the typical values of the chemical potential involved. The SB expressions are then well approximated by their zero-temperature limits, which satisfy the relation $\varepsilon_{\mathrm{SB}}^{0}=3 p_{\mathrm{SB}}^{0}=\frac{3}{4} \mu_{B} n_{\mathrm{SB}}^{0}$. The SB-normalized thermodynamic quantities (denoted by a bar) thus satisfy the identity

$$
\bar{\varepsilon} \approx-\frac{1}{3} \bar{p}+\frac{4}{3} \bar{n}_{B}+\frac{T s}{\varepsilon_{\mathrm{SB}}},
$$

where the approximation sign refers to the zerotemperature limit of the SB values. Using this identity, one may from the values of $\bar{p}, \bar{n}_{B}, \bar{\varepsilon}$ given in [16] determine the purely thermal component of the energy density, represented by the entropy. One thus finds that $T s / \varepsilon_{\mathrm{SB}}$ is essentially zero for $\mu_{B} / m_{\pi} \gtrsim 3$ [43], but the sharp peak already present in $\bar{\varepsilon}$, of course, remains. We are thus led to the conclusion that the high peak in the energy density is due to thermal excitations.

In order to see how this can come about, let us observe that the diquark dispersion relation below the onset of $\mathrm{BEC}$ is given by $E_{\boldsymbol{k}}=\sqrt{\boldsymbol{k}^{2}+m_{\pi}^{2}}-\mu_{B}$. Right at the BEC transition point, $\mu_{B}=m_{\pi}$, the dispersion is therefore quadratic at low momentum, and it still remains rather 
flat even when the chemical potential increases above $m_{\pi}$. Moreover, in the whole diquark condensation phase there is a massless NG boson stemming from the spontaneous breaking of the exact global symmetry associated with the conservation of the baryon number. As soon as the temperature is nonzero, the NG states will be abundantly populated, giving a large entropy, contributing in turn to the energy density. Whether this mechanism is physically correct can easily be tested by changing the temperature. If the peak in the energy density is really mostly due to thermal excitations, its height should be very sensitive to the changes in temperature. On the other hand, the peaks in the pressure and baryon density, not getting a contribution from the entropy, should be rather robust, being dominated by the condensate contribution.

With this physical picture in mind, it is now also clear how our model calculations should be modified in order to take the NG excitations in the account. In the linear sigma model or chiral perturbation theory, one must include loop effects which introduce the finite temperature into the system via the Matsubara summation. Within the NJL model, the mean-field approximation only includes quasifermionic modes. In order that the NG excitations be properly taken into account, one must, again, go beyond the mean-field approximation. Although both these extensions are in principle straightforward (the next-to-leading order results within chiral perturbation theory being even already available [6]), albeit maybe somewhat tedious, they may still not yield the desired quantitative agreement with the lattice simulations. Possible reasons are discussed in the concluding section.

\section{CONCLUSIONS}

In this paper we investigated the thermodynamics of two-color QCD with two light quark flavors. We devised a general method to construct interaction Lagrangians of the NJL type with the extended global SU(4) symmetry. Using the mean-field approximation, we then determined the phase diagram in the $\left(\mu_{B}, \mu_{I}\right)$ plane, including the FF pairing and the isospin splitting of the flavor chiral condensates.

One should emphasize that, since we only considered the chiral NJL model, confinement effects are not taken into account in our results. This can be done phenomenologically by adding the Polyakov loop as an additional degree of freedom [20]. However, this will only affect the physics at nonzero temperature, since the Polyakov loop NJL model reduces to pure NJL at zero temperature. Hence, the results of Sec. IV will be unaffected. Likewise, Figures [3, 4, and 5] will not change qualitatively; basically the only difference will be a rescaling of the critical temperatures to higher values.

Finally, in Sec. VI we discussed the thermodynamics of diquark BEC with a particular attention to available lattice data. We suggested that a proper inclusion of thermal NG excitations is crucial in order to achieve a quantitative agreement with them. While it would be very nice to pursue our model calculations as far as possible, we have some reservations as to whether this would lead to the desired goal. The reason is that the lattice computations were performed with rather heavy quarks. Consequently, the lightest non-NG bosonic state is the rho meson with $m_{\pi} / m_{\rho} \approx 0.8$. We see that there is no clear separation of scales, and therefore the success of any approach based on chiral symmetry and its spontaneous breaking is a priori questionable. One obvious remedy to this situation would be to perform lattice simulations with lighter quarks, but this may be technically rather demanding. On the other hand, one might try to avoid using chiral models by resorting to first-principle QCD calculations, based, e.g., on the Dyson-Schwinger equations 44] or the functional renormalization group approach [45].

Summarizing, we would like to stress once more that two-color QCD is a system whose study may teach about deconfinement in cold dense matter as well as other aspects of the real world. At present, the techniques that would allow us to investigate the phase diagram of twocolor QCD in detail are on the market. We believe that our future efforts will contribute to its general understanding.

\section{ACKNOWLEDGMENTS}

The authors are grateful to H. Abuki, K. Fukushima, S. Hands, X.-g. Huang, A. Maas, D. Nicmorus, D. Parganlija, and $\mathrm{H}$. Warringa for useful discussions and correspondence. The hospitality of the Niels Bohr International Academy where this project was initiated is gratefully appreciated. Part of the work was carried out during the stay of T.B. at the Norwegian University of Science and Technology, Trondheim. The research of T.B. was supported in part by the Alexander von Humboldt Foundation, and by the ExtreMe Matter Institute EMMI in the framework of the Helmholtz Alliance Program of the Helmholtz Association (HA216/EMMI). Numerical calculations were performed using the facilities of the Frankfurt Center for Scientific Computing.

\section{APPENDIX: THERMODYNAMIC POTENTIAL FOR FULDE-FERRELL PAIRING}

Consider for simplicity a nonrelativistic theory of two fermion species, $\psi_{\uparrow}, \psi_{\downarrow}$, defined by the (Euclidean) Lagrangian

$$
\mathscr{L}=\sum_{\sigma=\uparrow, \downarrow} \psi_{\sigma}^{\dagger}\left(\partial_{\tau}-\frac{\nabla^{2}}{2 m}-\hat{\mu}\right) \psi_{\sigma}-g \psi_{\uparrow}^{\dagger} \psi_{\downarrow}^{\dagger} \psi_{\downarrow} \psi_{\uparrow},
$$

where $g$ is the bare (attractive) coupling and $\hat{\mu}$ the diagonal chemical potential matrix in the flavor space. Introducing a collective field $\phi \sim \psi_{\downarrow} \psi_{\uparrow}$, performing the 
Hubbard-Stratonovich transformation, integrating out the fermions, and finally summing over the Matsubara frequencies, one arrives at an expression for the meanfield thermodynamic potential analogous to Eq. (6),

$$
\begin{aligned}
\Omega[\phi]= & \int d^{3} \boldsymbol{x} \frac{|\phi(\boldsymbol{x})|^{2}}{g} \\
& -\operatorname{Tr}\left\{\frac{1}{2} \mathscr{H}(\boldsymbol{x})+T \log \left[1+e^{-\beta \mathscr{H}(\boldsymbol{x})}\right]\right\},
\end{aligned}
$$

where

$$
\mathscr{H}(\boldsymbol{x})=\left(\begin{array}{cc}
-\frac{\boldsymbol{\nabla}^{2}}{2 m}-\mu_{\uparrow} & -\phi(\boldsymbol{x}) \\
-\phi^{*}(\boldsymbol{x}) & \frac{\boldsymbol{\nabla}^{2}}{2 m}+\mu_{\downarrow}
\end{array}\right),
$$

is the matrix Hamiltonian in the Nambu space, $\left(\psi_{\uparrow}, \psi_{\downarrow}^{\dagger}\right)$, and the trace operation is to be performed in the operator sense.

So far, no assumption on the particular form of the mean field configuration $\phi(\boldsymbol{x})$ was made; in principle this is to be determined by minimization of the functional $\Omega[\phi]$. Once again, however, Eq. (11) is only formal because $\operatorname{Tr} \mathscr{H}(\boldsymbol{x})$ is badly divergent. Subtracting the same expression for the normal phase (that is, $\phi=0$ ) at zero temperature with the corresponding Hamiltonian $\mathscr{H}_{0}$, one obtains the regularized thermodynamic potential as

$$
\begin{aligned}
\Omega_{\text {sub }}[\phi]= & \int d^{3} \boldsymbol{x} \frac{|\phi(\boldsymbol{x})|^{2}}{g}-\operatorname{Tr}\left\{\frac{1}{2}\left[\mathscr{H}(\boldsymbol{x})-\left|\mathscr{H}_{0}\right|\right]\right. \\
& \left.+T \log \left[1+e^{-\beta \mathscr{H}(\boldsymbol{x})}\right]\right\} .
\end{aligned}
$$

Within the nonrelativistic theory this prescription can be shown to remove all divergences once the coupling $g$ is renormalized. (In practice this is usually done by trading $g$ for the gap at zero temperature which is physically observable and thus free of divergences.)

Unfortunately, it is impossible to evaluate the trace of the $\log$ term for a general field configuration $\phi(\boldsymbol{x})$. The FF pairing corresponds to the Ansatz $\phi(\boldsymbol{x})=\Delta e^{2 i \boldsymbol{q} \cdot \boldsymbol{x}}$. Now the Hamiltonian may already be diagonalized. To this end, we perform the unitary transformation, $\mathscr{H} \rightarrow$ $\mathscr{H}^{\prime}=U_{\boldsymbol{q}}^{\dagger} \mathscr{H} U_{\boldsymbol{q}}$, where $U_{\boldsymbol{q}}=\operatorname{diag}\left(e^{i \boldsymbol{q} \cdot \boldsymbol{x}}, e^{-i \boldsymbol{q} \cdot \boldsymbol{x}}\right)$ and

$$
\mathscr{H}^{\prime}=\left(\begin{array}{cc}
\frac{(-i \boldsymbol{\nabla}+\boldsymbol{q})^{2}}{2 m}-\mu_{\uparrow} & -\Delta \\
-\Delta & -\frac{(-i \boldsymbol{\nabla}-\boldsymbol{q})^{2}}{2 m}+\mu_{\downarrow}
\end{array}\right) .
$$

The eigenvalues of this Hamiltonian are now easily found by Fourier transforming to momentum space. They are $E_{\boldsymbol{k}}^{-}$and $-E_{\boldsymbol{k}}^{+}$, where $E_{\boldsymbol{k}}^{ \pm}=E_{\boldsymbol{k}} \pm \delta \mu_{\boldsymbol{q}}, \delta \mu_{\boldsymbol{q}}=\delta \mu-(\boldsymbol{k}$. $\boldsymbol{q}) / m, E_{\boldsymbol{k}}=\sqrt{\xi_{\boldsymbol{k}}^{2}+\Delta^{2}}, \xi_{\boldsymbol{k}}=\left(\boldsymbol{k}^{2}+\boldsymbol{q}^{2}\right) / 2 m-\bar{\mu}$, and we have defined $\bar{\mu}=\left(\mu_{\uparrow}+\mu_{\downarrow}\right) / 2$ and $\delta \mu=\left(\mu_{\uparrow}-\mu_{\downarrow}\right) / 2$. Our crucial observation is that since the trace in (12) has a well defined mathematical meaning in combination with $\mathscr{H}_{0}$, we have to do the same unitary transformation $U_{\boldsymbol{q}}$ on it as well. The thermodynamic potential density therefore becomes

$$
\begin{aligned}
\frac{\Omega(\Delta, q)}{V}= & \frac{\Delta^{2}}{g}-\int \frac{d^{3} \boldsymbol{k}}{(2 \pi)^{3}}\left\{E_{\boldsymbol{k}}-\frac{1}{2}\left[\left|\xi_{\boldsymbol{k}}+\delta \mu_{\boldsymbol{q}}\right|\right.\right. \\
& \left.\left.+\left|\xi_{\boldsymbol{k}}-\delta \mu_{\boldsymbol{q}}\right|\right]+T \sum_{ \pm} \log \left[1+e^{-\beta\left(E_{\boldsymbol{k}} \pm \delta \mu_{\boldsymbol{q}}\right)}\right]\right\} .
\end{aligned}
$$

Note that subtracting naively the contribution of the normal phase from Eq. (11) would amount to replacing the second term in the curly brackets with $\frac{1}{2}\left[\left|\xi_{\boldsymbol{k}}+\delta \mu\right|+\mid \xi_{\boldsymbol{k}}-\right.$ $\delta \mu \mid]$. The difference is finite, but the naive subtraction would lead to an extra term in the thermodynamic potential, independent of $\Delta$ and going to $-\infty$ at large $\boldsymbol{q}$. Such a thermodynamic potential would not even have a minimum. Our derivation makes sure that the thermodynamic potential is both well defined and physically consistent.

In relativistic systems the same argument can be used, but the situation is less simple. The reason is that the divergences in the relativistic theory are more severe due to different quasiparticle dispersion relation, which is already signalized by the presence of a quadratic divergence $\sim \Lambda^{2} q^{2}$. The divergences are not completely removed by the subtraction of the normal phase and renormalization of the coupling constant. This means that the subtraction procedure remains somewhat ambiguous and is to be understood as a part of the definition of the model. This is, however, nothing new in the NJL model where one often handles divergent quantities in a rather formal manner [19]. Our strategy is as follows. We define the naive thermodynamic potential, $\Omega(\sigma, \rho, \Delta(\boldsymbol{x}))$, analogously to Eq. (11), and rewrite it as

$$
\Omega(\sigma, \rho, \Delta(\boldsymbol{x}))=\Omega(\sigma, \rho, \Delta(\boldsymbol{x}))-\Omega(\sigma, \rho, 0)+\Omega(\sigma, \rho, 0) .
$$

The last term is the thermodynamic potential of the "normal phase" while in the difference of the first two terms the most severe divergences cancel. It is this difference that the unitary transformation $U_{\boldsymbol{q}}$ is applied to. For the FF pairing this procedure leads to Eq. (77). One should, however, observe that since the final expression is still mildly (logarithmically) divergent, the result depends on the choice of the term to subtract, which was already noted in [13].

Here we have subtracted the "normal phase" with respect to the diquark condensate, but with unchanged values of the chiral condensates. By dimensional analysis, the thermodynamic potential suffers from the following $\boldsymbol{q}$-dependent divergences: a quadratic divergence $\sim q^{2}$ and logarithmic divergences $\sim q^{2} \sigma^{2}, q^{2} \rho^{2}, q^{2} \mu_{B, I}^{2}, q^{2} \Delta^{2}$. All divergences but the very last one are canceled by our subtraction procedure. Fortunately, the remaining divergence $\sim q^{2} \Delta^{2}$ is rather mild because in the FF phase the gap $\Delta$ is numerically much smaller than in the BCS phase. 
[1] D. T. Son and M. A. Stephanov, Phys. Rev. Lett. 86, 592 (2001), arXiv:hep-ph/0005225

[2] J. B. Kogut, M. A. Stephanov, and D. Toublan, Phys. Lett. B464, 183 (1999), arXiv:hep-ph/9906346 J. B. Kogut, M. A. Stephanov, D. Toublan, J. J. M. Verbaarschot, and A. Zhitnitsky, Nucl. Phys. B582, 477 (2000), arXiv:hep-ph/0001171

[3] A. V. Smilga and J. J. M. Verbaarschot, Phys. Rev. D51, 829 (1995), arXiv:hep-th/9404031.

[4] R. Rapp, T. Schafer, E. V. Shuryak, and M. Velkovsky, Phys. Rev. Lett. 81, 53 (1998), arXiv:hep-ph/9711396

[5] K. Splittorff, D. T. Son, and M. A. Stephanov, Phys. Rev. D64, 016003 (2001), arXiv:hep-ph/0012274.

[6] K. Splittorff, D. Toublan, and J. J. M. Verbaarschot, Nucl. Phys. B620, 290 (2002), arXiv:hep-ph/0108040. B639, 524 (2002), arXiv:hep-ph/0204076

[7] J. Wirstam, Phys. Rev. D62, 045012 (2000), arXiv:hep-ph/9912446 J. T. Lenaghan, F. Sannino, and K. Splittorff, D65, 054002 (2002), arXiv:hep-ph/0107099 J. Wirstam, J. T. Lenaghan, and K. Splittorff, D67, 034021 (2003), arXiv:hep-ph/0210447.

[8] B. Vanderheyden and A. D. Jackson, Phys. Rev. D64, 074016 (2001), arXiv:hep-ph/0102064, B. Klein, D. Toublan, and J. J. M. Verbaarschot, D72, 015007 (2005), arXiv:hep-ph/0405180, T. Kanazawa, T. Wettig, and N. Yamamoto(2009), arXiv:0912.4999 [hep-ph]

[9] T. Kanazawa, T. Wettig, and N. Yamamoto, J. High Energy Phys. 08, 003 (2009), arXiv:0906.3579 [hep-ph]

[10] L. A. Kondratyuk, M. M. Giannini, and M. I. Krivoruchenko, Phys. Lett. B269, 139 (1991).

[11] C. Ratti and W. Weise, Phys. Rev. D70, 054013 (2004), arXiv:hep-ph/0406159

[12] G.-f. Sun, L. He, and P. Zhuang, Phys. Rev. D75, 096004 (2007), arXiv:hep-ph/0703159

[13] K. Fukushima and K. Iida, Phys. Rev. D76, 054004 (2007), arXiv:0705.0792 [hep-ph].

[14] J. Polonyi and K. Szlachanyi, Phys. Lett. B110, 395 (1982); Y. Nishida, K. Fukushima, and T. Hatsuda, Phys. Rept. 398, 281 (2004), arXiv:hep-ph/0306066 S. Chandrasekharan and F.-J. Jiang, Phys. Rev. D74, $014506 \quad$ (2006), arXiv:hep-lat/0602031 K. Fukushima, J. High Energy Phys. 07, 083 (2008), arXiv:0806.1104 [hep-ph]

[15] S. Hands, I. Montvay, S. Morrison, M. Oevers, L. Scorzato, and J.-I. Skullerud, Eur. Phys. J. C17, 285 (2000), arXiv:hep-lat/0006018 J. B. Kogut, D. K. Sinclair, S. J. Hands, and S. E. Morrison, Phys. Rev. D64, 094505 (2001), arXiv:hep-lat/0105026. S. Hands, I. Montvay, L. Scorzato, and J.I. Skullerud, Eur. Phys. J. C22, 451 (2001), arXiv:hep-lat/0109029 J. B. Kogut, D. Toublan, and D. K. Sinclair, Phys. Rev. D68, 054507 (2003), arXiv:hep-lat/0305003 J.-I. Skullerud, S. Ejiri, S. Hands, and L. Scorzato, Prog. Theor. Phys. Suppl. 153, 60 (2004), arXiv:hep-lat/0312002 M. D'Elia, S. Conradi, and A. D'Alessandro, PoS LAT2006, 131 (2006), arXiv:hep-lat/0609057

[16] S. Hands, S. Kim, and J.-I. Skullerud, PoS LAT2005, 149 (2006), arXiv:hep-lat/0508027, Eur. Phys. J. C48,
193 (2006), arXiv:hep-lat/0604004, S. Hands, S. Kim, and J.-I. Skullerud(2010), arXiv:1001.1682 [hep-lat]

[17] T. Brauner, Mod. Phys. Lett. A21, 559 (2006), arXiv:hep-ph/0601010

[18] The particle content of our model Lagrangian is minimal compatible with the symmetry of two-color QCD. In contains only the modes which can be obtained from the ground state by a symmetry transformation, and are guaranteed to exist by the Goldstone theorem.

[19] S. P. Klevansky, Rev. Mod. Phys. 64, 649 (1992).

[20] T. Brauner, K. Fukushima, and Y. Hidaka, Phys. Rev. D80, 074035 (2009), arXiv:0907.4905 [hep-ph]

[21] C. Vafa and E. Witten, Phys. Rev. Lett. 53, 535 (1984).

[22] M. Buballa, Phys. Rept. 407, 205 (2005), arXiv:hep-ph/0402234

[23] M. G. Alford, J. A. Bowers, and K. Rajagopal, Phys. Rev. D63, 074016 (2001), arXiv:hep-ph/0008208 J. A. Bowers, J. Kundu, K. Rajagopal, and E. Shuster, D64, 014024 (2001), arXiv:hep-ph/0101067

[24] R. Casalbuoni and G. Nardulli, Rev. Mod. Phys. 76, 263 (2004), arXiv:hep-ph/0305069

[25] M. G. Alford, A. Schmitt, K. Rajagopal, and T. Schafer, Rev. Mod. Phys. 80, 1455 (2008), arXiv:0709.4635 [hep-ph]; Q. Wang(2009), arXiv:0912.2485 [nucl-th]

[26] E. V. Gorbar, M. Hashimoto, and V. A. Miransky, Phys. Lett. B632, 305 (2006), arXiv:hep-ph/0507303 E. V. Gorbar, M. Hashimoto, V. A. Miransky, and I. A. Shovkovy, Phys. Rev. D73, 111502 (2006), arXiv:hep-ph/0602251

[27] P. F. Bedaque and T. Schafer, Nucl. Phys. A697, 802 (2002), arXiv:hep-ph/0105150 D. B. Kaplan and S. Reddy, Phys. Rev. D65, 054042 (2002), arXiv:hep-ph/0107265

[28] T. Schafer, Phys. Rev. Lett. 96, 012305 (2006), arXiv:hep-ph/0508190 A. Kryjevski, Phys. Rev. D77, 014018 (2008), arXiv:hep-ph/0508180

[29] The fact that pions, unlike the diquark, form an isospin triplet is immaterial. In fact, any nonzero value of $\mu_{I}$ breaks the isospin symmetry explicitly to its $\mathrm{U}(1)_{I_{3}}$ subgroup generated by the third component of isospin, which the chemical potential is associated with. The charged pions then carry one unit of this unbroken charge, very much like the diquark carries baryon number.

[30] P. Fulde and R. A. Ferrell, Phys. Rev. 135, A550 (1964).

[31] A. I. Larkin and Y. N. Ovchinnikov, Sov. Phys. JETP 20, 762 (1965).

[32] J. A. Bowers and K. Rajagopal, Phys. Rev. D66, 065002 (2002), arXiv:hep-ph/0204079 K. Rajagopal and R. Sharma, D74, 094019 (2006), arXiv:hep-ph/0605316

[33] S. Matsuo, S. Higashitani, Y. Nagato, and K. Nagai, J. Phys. Soc. Jpn. 67, 280 (1998); D. Nickel and M. Buballa, Phys. Rev. D79, 054009 (2009), arXiv:0811.2400 [hep-ph].

[34] L. He, M. Jin, and P. Zhuang, Phys. Rev. D74, 036005 (2006), arXiv:hep-ph/0604224 X.-g. Huang, Q. Wang, and P.-f. Zhuang, Int. J. Mod. Phys. E17, 1906 (2008), arXiv:0710.3634 [hep-ph]

[35] E. V. Gorbar, M. Hashimoto, and V. A. Mi-

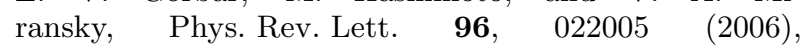
arXiv:hep-ph/0509334 
[36] D. M. Eagles, Phys. Rev. 186, 456 (1969); A. J. Leggett, J. Phys. Colloques 41, 19 (1980).

[37] A. Sedrakian and D. H. Rischke, Phys. Rev. D80, 074022 (2009), arXiv:0907.1260 [nucl-th].

[38] D. J. Thouless, Ann. Phys. (N.Y.) 10, 553 (1960).

[39] S. Hands, P. Sitch, and J.-I. Skullerud, Phys. Lett.B662, 405 (2008), arXiv:0710.1966 [hep-lat]

[40] Y. Nishida and H. Abuki, Phys. Rev. D72, 096004 (2005), arXiv:hep-ph/0504083 H. Abuki, Nucl. Phys. A791, 117 (2007), arXiv:hep-ph/0605081; M. Kitazawa, D. H. Rischke, and I. A. Shovkovy, Phys. Lett. B663, 228 (2008), arXiv:0709.2235 [hep-ph]

[41] In order to minimize the cutoff artifacts in the NJL model calculation, we evalute the SB thermodynamic quantities using the same model with the cutoff, setting the constituent quark mass as well as other condensates to zero by hand. Using this prescription, the SB-normalized expressions go to one - as they should - in the limit of large chemical potential and/or temperature.
[42] D. K. Campbell, R. F. Dashen, and J. T. Manassah, Phys. Rev. D12, 979 (1975); L.-y. He, M. Jin, and P.-f. Zhuang, D71, 116001 (2005), arXiv:hep-ph/0503272

[43] This suggests that the Karsch coefficient, that renormalizes the energy density without affecting the pressure and baryon density and is independent of the chemical potential [16], should not be much smaller than one. Otherwise, the entropy would come out negative at high chemical potential, which would, of course, be unphysical.

[44] P. Maris, C. D. Roberts, and S. M. Schmidt, Phys. Rev. C57, 2821 (1998), arXiv:nucl-th/9801059 D. Nickel, J. Wambach, and R. Alkofer, D73, 114028 (2006), arXiv:hep-ph/0603163 T. Klahn, C. D. Roberts, L. Chang, H. Chen, and Y.-X. Liu(2009), arXiv:0911.0654 [nucl-th]

[45] J. Berges, D.-U. Jungnickel, and C. Wetterich, Eur. Phys. J. C13, 323 (2000), arXiv:hep-ph/9811347, B.-J. Schaefer and J. Wambach, Phys. Part. Nucl. 39, 1025 (2008), arXiv:hep-ph/0611191. 